\title{
Prise En Charge Des Péritonites Aiguës Dans Un Hôpital De District En Afrique Sub-saharienne : Cas Du Bénin
}

\author{
Bio Tamou Sambo \\ Salako Alexandre Allodé \\ Service de Chirurgie Générale, CHU de Parakou, \\ Université de Parakou, Bénin \\ Didier Sewadé Wekpon
}

Service de Chirurgie Générale, Hôpital de district de Bembéréké, Bénin

\author{
Djifid Morel Séto \\ Montcho Adrien Hodonou
}

Service de Chirurgie Générale, CHU de Parakou,

Université de Parakou, Bénin

\section{Babatoundé Dossou}

Service de Chirurgie Générale, Hôpital de district de Bembéréké, Bénin

Doi: 10.19044/esj.2017.v13n36p388 URL:http://dx.doi.org/10.19044/esj.2017.v13n36p388

\begin{abstract}
Introduction: Peritonitis remains a public health problem in Africa. We aim to describe the epidemiological, etiological and therapeutic aspects of acute peritonitis in a district hospital in Sub Saharan Africa. Methods: This was a descriptive study with prospective data collection over a period of 15 months from May $1^{\text {st }} 2015$ to July $31^{\text {st }} 2016$ in Bembereke district hospital. It has taken into account all the patients managed in the general surgery department for acute generalized peritonitis that has been confirmed at laparotomy. Results: Fifty-three patients, 38 men (71.7 \%) and 15 women $(28.3 \%)$ had been registered. The average age of the patients was $19.8 \pm$ 16.9 years. The main etiologies were: non-traumatic ileal perforation from typhoid infection 52.8\%; perforated gastric or duodenal ulcer $17 \%$; complicated appendicitis and abdominal traumas $11.3 \%$ each one. Twenty nine patients $(54.7 \%)$ have been operated by a surgeon and the 24 remaining $(45.3 \%)$ by a general practitioner with surgical skills. Twenty one patients (39.6\%) had postoperative complications of which 11 cases of parietal suppurations $(52.4 \%)$. The mortality rate was $11.3 \%$. The mean hospital stay was $22.5 \pm 4$ days. Conclusion: In northern-Benin, peritonitis remains
\end{abstract}


dominated by the complications of typhoid fever. The mortality rate remains high. Prevention requires good hygiene and awareness of early consultations.

Keywords: Peritonitis, district hospital, typhic perforation

\section{Résumé}

Introduction: La péritonite demeure un problème de santé publique en Afrique. Notre but était de décrire les aspects épidémiologiques, étiologiques et thérapeutiques de la péritonite aigue généralisée dans un hôpital de zone en Afrique Sub-saharienne. Méthodologie: Il s'agissait d'une étude descriptive avec un recueil prospectif des données sur une période de 15 mois allant du $1^{\text {er }}$ Mai 2015 au 31Juillet 2016 à l'hôpital de zone de Bembéréké. Etaient inclus tous les patients pris en charge dans le service de chirurgie générale pour péritonite aigue généralisée confirmée à la laparotomie. Résultats: Cinquante trois patients, 38 de sexe masculin $(71,7 \%)$ et 15 de sexe féminin $(28,3 \%)$ avaient été retenus. L'âge moyen des patients était de 19,8 $\pm 16,9$ ans. Les principales étiologies étaient: la perforation iléale non traumatique d'origine typhique $52,8 \%$; la perforation d'ulcère gastrique ou duodénale $17 \%$; l'appendicite compliquée et les traumatismes abdominaux $11,3 \%$ chacun. Vingt neuf patients $(54,7 \%)$ avaient été opérés par un chirurgien et les 24 autres $(45,3 \%)$ par des médecins généralistes à compétence chirurgicale. Vingt un patients $(39,6 \%)$ avaient présenté des complications postopératoires dont 11 cas de suppuration pariétale $(52,4 \%)$. Le taux de mortalité était de $11,3 \%$. La durée moyenne d'hospitalisation était de 22,5 \pm 4 jours. Conclusion: Au nordBénin, la péritonite reste dominée par les complications de la fièvre typhoïde. Le taux de mortalité demeure élevé. La prévention passe par une bonne hygiène et une sensibilisation de la population pour des consultations précoces.

Mots-clés: Péritonite, hôpital de zone, perforation typhique

\section{Introduction}

La péritonite se définit comme une réponse inflammatoire de tout ou partie du péritoine à une agression dont l'origine est le plus souvent infectieuse (Monteiro et al. 2007). Elle demeure un problème de santé publique en Afrique. Elle est l'une des principales causes d'admission en réanimation et est associée à une mortalité élevée (Tchaou et al. 2014). Le pronostic de la péritonite est directement lié à la précocité du diagnostic et à la qualité de la prise en charge thérapeutique (Mariette et al. 2006). L'objectif de cette étude est de décrire les aspects épidémiologiques, 
étiologiques et thérapeutiques de la péritonite aigue généralisée (PAG) dans un hôpital de zone.

\section{Matériels et méthodes}

Il s'agissait d'une étude prospective transversale descriptive sur une période de 15 mois allant du $1^{\mathrm{er}}$ mai 2015 au 31 juillet 2016. Etaient inclus, tous les patients admis dans le service de chirurgie générale de l'hôpital de zone de Bembéréké (HZB) et chez qui le diagnostic de péritonite aigue généralisée avait été confirmé à la laparotomie. L'HZB est le premier niveau de référence des communes de Bembéréké et Sinendé au nord du Bénin. Le centre de référence de $2^{\text {ème }}$ niveau est à Parakou à $110 \mathrm{~km}$. Les patients avaient été opérés par un chirurgien ou un médecin généraliste à compétence chirurgicale formé sur le tas. La réanimation pré opératoire était assurée par l'opérateur en collaboration avec un infirmier anesthésiste. Cette réanimation était poursuivie en per et post opératoire. Le bilan pré opératoire en urgence était limité et comprenait un groupage sanguin rhésus, une numération formule sanguine, une glycémie et la créatininémie. La coproculture et l'hémoculture n'étaient pas disponibles. Le sérodiagnostic de Widal et Félix avait été réalisé chez tous les patients qui avaient une perforation iléale non traumatique. L'origine typhique de la perforation était basée sur les arguments épidémiologiques de la fièvre typhoïde, les données biologiques et cliniques, le siège et l'aspect des perforations, la présence de ganglions mésentériques. Certains patients avaient bénéficié de la radiographie de l'abdomen sans préparation (ASP) ou de l'échographie abdominale. Les données recueillies ont été analysées avec les logiciels Epi info et SPSS.

\section{Résultats}

\section{Données épidémiologiques}

Cinquante trois cas de PAG avaient été recensés. Durant la période d'étude, 2254 patients avaient été opérés dont 80 pour des urgences chirurgicales abdominales (UCA). La PAG représentait 2,3\% des activités du bloc opératoire et $66,2 \%$ des UCA. La fréquence hospitalière était de 3,5 cas par mois. L'âge moyen des patients était de 19,8 $\pm 16,9$ ans avec des extrêmes de 4 et 71 ans. La tranche d'âge de 5 à 15 ans était la plus représentée avec 22 cas soit $41,5 \%$. Il y avait 38 patients de sexe masculin $(71,7 \%)$ et 15 de sexe féminin $(28,3 \%)$ soit une sex-ratio de 2,5 .

\section{Données étiologiques}

Chez deux patients $(3,8 \%)$, aucune source de contamination n'avait été retrouvée. La péritonite était secondaire pour les 51 autres cas (96,2\%). Elle était communautaire dans tous les cas. Les étiologies de ces péritonites secondaires étaient par ordre décroissant: 
- $\quad$ Les perforations supposées d'origine typhique dans 28 cas (52,8\%), - $\quad$ Les perforations d'ulcère gastrique ou duodénal 9 cas $(17 \%)$ à raison de 6 cas de perforation gastrique et de 3 cas de perforation duodénale,

- $\quad$ Les appendicites compliquées 6 cas $(11,3 \%)$,

- $\quad$ Les traumatismes abdominaux 6 cas $(11,3 \%)$. Il s'agissait dans tous les cas des perforations iléales.

- $\quad$ Les infections génitales 2 cas $(3,8 \%)$

\section{Données thérapeutiques}

Selon la classification ASA (American Society of Anesthesiologists), 45 patients $(84,9 \%)$ étaient classés ASA 2 et les 8 restants $(15,1 \%)$ ASA3. Quarante quatre patients (83\%) avaient eu un traitement avant leur admission à l'HZB. Parmi eux, 21 patients $(47,3 \%)$ avaient reçu un traitement médical dans un autre centre de santé, 11 patients $(25 \%)$ avaient fait de l'automédication et 12 autres $(27,3 \%)$ avaient eu recours à un traitement traditionnel. Vingt neuf patients $(54,7 \%)$ avaient été opérés par un chirurgien et $24(45,3 \%)$ par des médecins généralistes à compétence chirurgicale. Le lavage de la cavité abdominale était systématique chez tous les malades tandis que le drainage avait été réalisé dans 48 cas $(90,6 \%)$. Les gestes chirurgicaux posés sont consignés dans le tableau 1.

Nous avons enregistré 21 cas de complications soit une morbidité postopératoire de $39,6 \%$. Il s'agissait de: suppuration pariétale 11 cas $(52,4 \%)$, éviscération 4 cas $(19 \%)$, fistule stercorale 3 cas $(14,3 \%)$, péritonite postopératoire 2 cas $(9,5 \%)$, septicémie 1 cas $(4,8 \%)$. Six patients $(11,3 \%)$ sont décédés: 2 cas de perforation iléale d'origine typhique, 2 cas de perforation duodénale et deux cas de perforation gastrique. La durée moyenne d'hospitalisation était de 21,7 74 jours avec les extrêmes de 1 et 91 jours.

Tableau 1: Répartition des gestes chirurgicaux posés en fonction de l'étiologie de la PAG Etiologies (fréquence)

Gestes chirurgicaux (nombre)

\begin{tabular}{|c|c|}
\hline $\begin{array}{c}\text { Péritonite par perforation supposée } \\
\text { typhique }(52,8 \%)\end{array}$ & $\begin{array}{l}\text { - Lavage-drainage de l'abdomen (28) } \\
\text { - Excision-suture iléale (16) } \\
\text { - Résection-anastomose (8) } \\
\text { - Iléostomie (4) }\end{array}$ \\
\hline $\begin{array}{l}\text { Péritonite par perforation gastrique ou } \\
\text { duodénale }(17 \%)\end{array}$ & $\begin{array}{l}\text { - Lavage-drainage de l'abdomen (9) } \\
\text {-Excision-suture gastrique ou duodénale (5) } \\
\text { - Excision-suture et omentoplastie (4) }\end{array}$ \\
\hline Péritonite appendiculaire $(11,3 \%)$ & $\begin{array}{l}\text { - Lavage-drainage de l'abdomen (5) } \\
\text { - Appendicectomie (6) }\end{array}$ \\
\hline
\end{tabular}




$\begin{array}{cc}\text { Péritonite post traumatique (11,3\%) } & \text { - Lavage-drainage de l'abdomen (4) } \\ & \text { - Lavage de l'abdomen (2) } \\ \text { - Excision-suture iléale (6) }\end{array}$

Péritonite génitale $(3,8 \%)$

- Lavage-drainage de l'abdomen (2)

- Excision-suture utérine (1)

Péritonite primitive $(3,8 \%)$

Lavage de l'abdomen (2)

\section{Discussion}

La PAG occupait la première place des UCA avec 66,2\%. Cette fréquence élevée est bien au dessus des valeurs rapportées dans d'autres séries africaines au Togo, au Burkina et au Niger (Kassegne et al. 2013 ; Ouangre et al. 2013 ; Harouna et al. 2001) où on retrouve 32,2 à $54,5 \%$. Le jeune âge des patients souffrant de PAG (moyenne de 19,8 ans dans notre série) est relevé par plusieurs auteurs avec une fourchette de 23 à 39,5 ans (Kambiré et al. 2017; Kassegne et al. 2016; Mehinto et al. 2010 ; Ngo Nonga et al. 2010) Cette affection constitue donc l'apanage des sujets jeunes et s'observe surtout chez les hommes avec un rapport Homme/femme qui varie entre 2,4 et 5 (Kassegne et al. 2013 ; Kambiré et al. 2017 ; Mehinto et al. 2010).

Les étiologies de la PAG varient d'un auteur à un autre comme le montre le tableau 2.

Tableau 2: Etiologies de la PAG selon différents auteurs

\begin{tabular}{ccc}
\hline Auteurs & Principale étiologie & Autres étiologies \\
\hline Notre série & $\begin{array}{c}\text { Perforation iléale non } \\
\text { traumatique d'origine typhique } \\
52,8 \%\end{array}$ & $\begin{array}{c}\text { Perforation d'ulcère gastrique ou } \\
\text { duodénal } 17 \%\end{array}$ \\
& $\begin{array}{c}\text { Complication d'appendicite } 11,3 \% \\
\text { Traumatisme abdominal } 11,3 \%\end{array}$ \\
$\begin{array}{c}\text { Kassegne I } \\
\text { (Togo) [4] }\end{array}$ & $\begin{array}{c}\text { Perforation iléale non } \\
\text { traumatique 64,2\% }\end{array}$ & $\begin{array}{c}\text { Complication d'appendicite 16\% } \\
\text { Origine pelvienne 3,1\% }\end{array}$ \\
$\begin{array}{c}\text { Kambiré JL } \\
\text { (Burkina-Faso) }\end{array}$ & $\begin{array}{c}\text { Perforation d'ulcère gastriques } \\
\text { [7] }\end{array}$ & $\begin{array}{c}\text { Perforation iléale non traumatique } \\
27 \%\end{array}$ \\
$\begin{array}{c}\text { Ngo Nonga A } \\
\text { (Cameroun) [10] }\end{array}$ & $\begin{array}{c}\text { Perforation d'ulcère gastriques } \\
\text { et duodénaux 32\% }\end{array}$ & $\begin{array}{c}\text { Complication d'appendicite 17\% } \\
\text { Perforation digestive non } \\
\text { traumatique 11\% }\end{array}$ \\
& & Origine pelvienne 5,8\%
\end{tabular}


Dans notre série, la perforation iléale d'origine typhique était la cause la plus fréquente soit $52,8 \%$. En effet la population de la région de Bembéréké est essentiellement rurale. La précarité de l'hygiène et les difficultés d'accès à l'eau potable y constituent des facteurs favorisants de la fièvre typhoïde. En occident par contre, les péritonites sont principalement d'origine colique (sigmoïdites), vésiculaire et appendiculaire (Gauzit et al. 2009; Montravers et al. 2015).

Quarante quatre patients $(83 \%)$ avaient reçu un traitement avant l'admission dont $27,3 \%$ d'automédication et $25 \%$ de traitement traditionnel. Harouna et al. au Niger (Harouna et al. 2001) avaient mentionné dans leur étude que les patients faisaient souvent recours au traitement traditionnel. Ceci pourrait s'expliquer par la forte croyance culturelle et contribuerait au retard de la prise en charge adéquate. La morbidité était de 39,6\% avec en tête la suppuration pariétale $(52,4 \%)$. Le même constat avait été fait par d'autres auteurs en Afrique (Kassegne et al. 2013 ; Ngo Nonga et al. 2010) qui ont mentionné des taux de suppuration allant 21,6 à 59,3\%. Ceci peut être lié à l'état d'hygiène des patients, aux conditions d'asepsie parfois peu satisfaisantes et les interventions réalisées dans des situations d'urgence. Nous avons déploré six décès soit une mortalité de $11,3 \%$. Ce taux est proche de celui des séries d'Allodé et al. (Allodé et al. 2017) et de Kassigne et al. (Kassegne et al. 2013) qui était respectivement de $14,5 \%$ et $14,8 \%$. Une étude en France avait retrouvé 15\% de taux de mortalité malgré le plateau technique de qualité (Gauzit et al. 2009). Selon Montravers (Montravers et al. 2015), la mortalité des PAG dépend en fait de l'état du patient, de la précocité de la prise en charge mais également de l'étiologie. A cet effet Mehinto et al. (Mehinto et al. 2010) ont obtenu une mortalité nulle pour les PAG d'origine typhique. La durée moyenne d'hospitalisation des patients était de 21,7 jours dans notre étude pendant que Mehinto et al. à Cotonou (Mehinto et al. 2010) ont relevé 12 jours et Mabewa et al. en Tanzanie (Mabewa et al. 2015) mieux encore (7 jours). Le séjour postopératoire a été long dans notre série du fait de la gestion des nombreuses complications survenues.

\section{Conclusion}

La PAG demeure une affection fréquente et grave au nord du Bénin. La sensibilisation de la population sur l'hygiène et l'approvisionnement en eau potable par les autorités contribuerait à une réduction de la prévalence de la fièvre typhoïde et donc de la PAG.

\section{Déclaration de conflits d'intérêt}

Les auteurs déclarent n'avoir aucun conflit d'intérêt en rapport avec cet article. 


\section{References:}

1. Monteiro, MC., Danielou, A., Piemont, Y., Hansmann, Y., \& Rohr, S. (2007). Prélèvements microbiologiques et traitements antibiotiques probabilistes des péritonites secondaires communautaires. J Chir ; 144:486-91.

2. Tchaou, BA., Assouto, P., Laine, JAA., \& Chobli, M. (2014). Gravité et pronostic des péritonites aiguës généralisées admises en service de réanimation. Méd Afr Noire ; 6110:507-13.

3. Mariette, C. (2006). Principes de prise en charge chirurgicale des péritonites postopératoires. Journal de Chirurgie ; 143(2):84-7.

4. Kassegne, I., Kanassoua, KK., Sewa, EVB., Sambiani, DM., Ayite, AE., \& al. (2013). Prise en charge des péritonites aiguës généralisées au Centre Hospitalier Universitaire de Kara. Saranf ; 18(2):115-21.

5. Ouangre, E., Zida, M., Bonkoungou, PG., Sanou, A., \& Traoré, SS. (2013). Les péritonites aigües généralisées en milieu rural au Burkina Faso : a propos de 221 cas, Rev. Cames Sante ; 1(2):75-9.

6. Harouna, YD., Abdou, I., Saidou, B., \& Bazira, L. (2001). Les péritonites en milieu tropical : particularités étiologiques et facteurs pronostics actuels : à propos de 160 cas. Méd Afr Noire ; 48(3):1035.

7. Kambiré, JL., Zaré, C., Sanou, BG., \& Kambou, T. (2017). Étiologies et pronostic des péritonites secondaires au centre hospitalier universitaire de Bobo-Dioulasso (Burkina Faso) Journal Africain d'Hépato-Gastroentérologie ;10(2):119-23.

8. Kassegne, I., Sewa, EV., Kanassoua, KK., Alassani, F., Adabra, K., Amavi, AK., \& al. (2016). Aspects diagnostiques, thérapeutiques et pronostiques des perforations typhiques du grêle à Dapaong, Togo Med Sante Trop ; 26:71-4.

9. Mehinto, DK., Gandaho, I., Adoukonou, O., Bagnan, OK., \& Padonou, N. (2010). Aspects épidémiologiques, diagnostiques et thérapeutiques des perforations du grêle d'origine typhique en chirurgie viscérale du Centre National Hospitalier et UniversitaireHubert Koutoucou Maga de Cotonou. Méd Afr Noire ; 57(11):53540.

10. Ngo Nonga, B., MouafoTambo, FF., Ngowe Ngowe, M., Takongmo, S., \& Sosso, MA. (2010). Etiologies des péritonites aigues généralisées au CHU de Yaoundé. Rev.Afr.Chir.Spéc ; 4(7):30-2.

11. Gauzit, R., Péan, Y., Barth, X., Mistretta, F., \& Lalaudi, O. (2009). Epidemiology, Management and Prognosis of secondary nonpostoperative peritonitis: A French prospective observational multicenter study. Surgical Infection ; 10(2):119-23. 
12. Montravers, P., Dupont, H., Leone, M., Constantin, JM., Mertes, PM. $\&$ al. (2015). Prise en charge des infections intra-abdominales SFAR ; 1(1):75-99.

13. Allode, AS., Dossou, FM., Hodonou, AM., Seto, M., Gbessi, GD., Tamou Sambo, B., \& Olory-Togbe, JL. (2017). Non traumatic intestinal perforation in the regional hospital Borgou of Benin. Epidemiological and therapeutic characteristics. Int Surg J; 4(4):1376-9.

14. Mabewa, A., Seni, J., Chalya, PL., Mshana, SE., \& Gilyoma, JM. (2015). Etiologies, treatment outcome and factors among patients with secondary peritonitis at Bugando Medical Centre, Mwanza, Tanzania World J Emerg Surg; 10:47. 